\title{
Hypoxis hemerocallidea alters metabolic parameters and hepatic histomorphology in streptozotocin- nicotinamide-induced diabetic male rats under antiretroviral therapy
}

\author{
Ismail Olasile Onanuga ${ }^{1,2}$, Ayoola Isaac Jegede ${ }^{1,3}$, Ugochukwu Offor ${ }^{1}$, Oluwatosin O. Ogedengbe ${ }^{1}$, \\ Edwin C.S. Naidu ${ }^{1}$, Anetkan I. Peter ${ }^{1}$, Onyemaechi Okpara Azu ${ }^{1,4}$
}

\author{
${ }^{1}$ Discipline of Clinical Anatomy, School of Laboratory Medicine and Medical Sciences, \\ Nelson R Mandela School of Medicine, University of KwaZulu-Natal, Durban, \\ South Africa \\ ${ }^{2}$ Department of Anatomy, Faculty of Biomedical Sciences, Kampala International \\ University, Dar es Salaam, Tanzania \\ ${ }^{3}$ Anatomy Department, Faculty of Basic Medical Sciences, College of Health Sciences, \\ Ladoke Akintola University of Technology, Ogbomoso, Nigeria \\ ${ }^{4}$ Department of Anatomy, School of Medicine, Windhoek, Namibia
}

Submitted: 4 April 2017

Accepted: 23 June 2017

Arch Med Sci 2020; 16 (1): 212-224

DOI: https://doi.org/10.5114/aoms.2018.75220

Copyright (c) 2018 Termedia \& Banach

\begin{abstract}
Introduction: Highly active antiretroviral therapy (HAART) and HIV/AIDS have been demonstrated to induce endocrine/metabolic dysfunction with a consequential increase in morbidity/mortality due to organ toxicities. This study aimed at investigating the possible protective effect of Hypoxis hemerocallidea $(H H)$ against metabolic and hepatic histomorphology of diabetic rats under HAART.

Material and methods: Sixty-two adult male Sprague-Dawley rats were divided into a normoglycemic group $A(n=6)$ and 7 diabetic $(110 \mathrm{mg} / \mathrm{kg}$ nicotinamide $+45 \mathrm{mg} / \mathrm{kg}$ streptozotocin) groups $(\mathrm{B}-\mathrm{H})(n=8)$ and treated according to protocols. Concomitant treatment with adjuvant $\mathrm{HH}$ and HAART resulted in the least \%body weight gain as the liver weight decreased in all treated animals.

Results: Significant changes in serum lipids were aggravated by treatment with $H H$ and HAART, triglycerides and total cholesterol levels were elevated $(p<0.001 / 0.05)$, but changes in high-density lipoprotein (HDL) and total protein levels were insignificant. While artherosclerotic and cardiopulmonary indexes remained insignificant, concomitant use of $\mathrm{HH}$ with HAART in diabetes resulted in reduction of low-density lipoprotein (LDL) $(p<0.001)$, and increased triglyceride $(p<0.05)$ and total cholesterol $(p<0.001)$. The parameters of liver injury showed a significant $(p<0.05)$ increase in ALT of animals treated with $\mathrm{HH}$ alone, HAART $+\mathrm{HH}$ and melatonin; however, an insignificant decline in AST level was recorded. Treatment with adjuvant HAART, $H H$ and melatonin resulted in significant $(p<0.005 / 0.0001)$ up-regulation of ALP and total bilirubin levels. Histopathology derangement ranged from severe hepatocellular distortions, necrosis with reduced glycogen expression following co-treatment of HAART+melatonin, $\mathrm{HH}$ and HAART alone in diabetes.

Conclusions: Presumptive hypoglycemic use of $H H$ with HAART by people living with HIV/AIDS requires caution as implications for hepatocellular injuries are suspected with further uncontrolled metabolic disorder.
\end{abstract}

\section{Corresponding author:}

Ismail Olasile Onanuga MSc University of KwaZulu-Natal 719 Umbilo Road 40000 Durban, South Africa Phone: +27846465847 E-mail: onanugaismail@ gmail.com

Key words: antiretroviral therapy, hepatic dysfunction, Hypoxis hemerocallidea, metabolic disorder, lipids. 


\section{Introduction}

The evolution and availability of highly active antiretroviral therapy (HAART) has translated into many positive consequences for the HIV widespread in sub-Saharan Africa. Not only with lifestyle changes, HAART has led to reduced morbidity and optimism that HIV can be managed similar to other chronic diseases [1]. This in turn has led to chronic complications of HIV in clinical practice [2]. The clear success of HAART in controlling HIV replication and restoring immunity has been tempered by the recognition that metabolic disorders and syndrome (MS) are increasing in incidence among people living with HIV (PLWHA). The World Health Organization (WHO) in 1999 defined MS as the combination of the presence of diabetes mellitus (DM), impaired fasting glucose, impaired glucose tolerance or insulin resistance, combined with two of the following criteria: waist-to-hip ratio $(\mathrm{WHR})>0.90$ in men, serum triglycerides $>150 \mathrm{mg} / \mathrm{dl}$ or high-density lipoprotein (HDL)-cholesterol $<35 \mathrm{mg} / \mathrm{dl}$ in men, urinary albumin excretion rate $>20 \mu \mathrm{g} / \mathrm{min}$ and blood pressure $>140 / 90 \mathrm{~mm} \mathrm{Hg}$ [3]. The prevalence of metabolic syndrome was $14 \%(n=114 ; 83$ men) according to International Diabetic Federation (IDF) criteria and $18 \%$ ( $n=139 ; 118$ men) according to Adult Treatment Panel III (ATPIII) criteria. Half of the patients (49\%) exhibited two or more features of metabolic syndrome but were not classified as having the syndrome because they had normal or low waist circumferences or waist-to-hip ratios. Metabolic syndrome was more common in those currently receiving protease inhibitors as five to nine higher prevalence of type 2 diabetes has been reported in those with metabolic syndrome [4].

Both HIV and HAART have been shown to induce endocrine or metabolic dysfunction [5] with a consequential increase in morbidity and mortality due to cardiovascular diseases [6]. The strongest relationship with diabetes was found for exposure to stavudine, while treatment with zidovudine and didanosine was also associated with an increased risk of diabetes [4, 7]. Some HAART, such as zidovudine and stavudine, have been associated with hepatic toxicity [8] with potential contribution partly to poor compliance and adherence which may necessitate switch of therapy. Studies in the recent past have also evaluated the utility of liver enzymes (alanine aminotransferase (ALT), aspartate aminotransferase (AST) and $\gamma$-glutamyl transpeptidase) in the management of HIV seropositive patients receiving HAART [9].

The management of HIV/AIDS is gradually expanding to include the chronic and metabolic complications of the disease, and the adverse effects associated with its treatments. The management choices of DM are similar to those in the HIV-negative or general population. Lifestyle modification and psychosocial support are a critical part of management of DM among HIV-infected patients. Endurance and resistance exercise have been shown to have positive effects on metabolic parameters in HIV-infected patients [10]. Where available, insulin is usually considered the primary line of therapy for newly diagnosed patients with severe hyperglycemia, especially in the presence of lipodystrophy syndrome or for patients with contraindications to oral anti-diabetic drugs (OADs). The high cost of insulin and HAART associated toxicities necessitates the need for safer and less demanding adjuvants. Plant-based adjuvants have the potential to fill this need. The search for herbal remedies has continued to grow due to their accessibility, relatively low cost and perceived effectiveness. Although their biologically active compounds are still unknown, herbal products or their extracts are widely used [11].

Hypoxis hemerocallidea $(H H)$, also known as Hypoxis rooperi (Hypoxidaceae), has a long history of traditional use for a variety of ailments [12] and more recently has been the subject of several scientific studies. In many parts of Africa, the corms of this attractive yellow flowered herb have been used in the treatment of urinary diseases, prostate hypertrophy, and internal cancer and more recently as an immune booster for people living with HIV-AIDS. HH is noted for the occurrence of a hypoxide which is a secondary metabolite of the plant that is hydrolyzed into rooperol in the large intestine, the active and powerful antioxidant component of the corm [13]. Pharmacokinetic studies have indicated that rooperol can be found in feces and metabolites are found in the serum and urine as its glycosides, sulfates, mixed glucuronides, and sulfuronides [14]. With increasing interest in the use of phytosterols (one of the phytochemical components of $H H$ ) for the reduction of serum cholesterol and for immune boosting, there has been a resultant increase in scientific investigations surrounding these benefits [15]. Interestingly, there has been a surge in commercially available herbal medicines containing sterols with $\mathrm{HH}$ extract enrichments claimed to be efficacious against a variety of diseases. However, the scientific validation of these claims remains to be verified despite its anti-inflammatory, antimicrobial, antidiabetic, anticonvulsant and anticancer properties reported by various authors [16, 17], and there is not any antiretroviral based therapy. Consequently, there is a scarcity of literature explaining its attenuating influence on the metabolic perturbations associated with HAART. However, studies from our laboratory [18] indicate that the perceived positive benefits of extracts from $\mathrm{HH}$ 
require further scientific investigation in view of the negative hepatotoxic indices emanating from a normoglycemic animal model.

Whilst PLWHA continue to patronize $\mathrm{HH}$ products for various purpose(s), there is a need to understand potential interactions and benefits following antiretroviral therapy in diabetic comorbidity. Therefore, this study investigated the metabolic roles of crude aqueous extracts of Hypoxis hemerocallidea $(H H)$ and its effects on morphological changes in the liver tissues of a diabetic experimental animal model following highly active antiretroviral therapy.

\section{Material and methods}

\section{Drugs and chemicals}

Nicotinamide, melatonin, lamivudine (3TC), zidovudine and nevirapine (Aspen) were sourced from Pharmed Ltd., Durban, South Africa. Streptozotocin (STZ) (Sigma-Aldrich, St. Louis, MO, USA), which was of analytical grade quality, was purchased from Capital Lab Supplies, Durban, South Africa. Glucose strips (Bayer Contour TS, Basel, Switzerland) was purchased from a local pharmacy.

\section{Collection of plant material}

Fresh corms of Hypoxis hemerocallidea were purchased from a local 'Muthi' in Umbilo Road, Durban, KwaZulu-Natal, between June and July, 2014. The corms were authenticated at the Department of Life Science, Westville Campus, University of KwaZulu-Natal, Durban, South Africa.

\section{Extraction of Hypoxis hemerocallidea}

Fresh Hypoxis corms were extracted according to the procedure of Ojewole et al. [19]. They were washed with water, cut into smaller pieces, air dried at room temperature $\left(25-28^{\circ} \mathrm{C}\right)$ and ground into powdered form in a commercial blender. The milled corm was soaked in hot distilled water and extracted twice, on each occasion with 2.5 I of hot distilled water (at $90-100^{\circ} \mathrm{C}$ ) for $12 \mathrm{~h}$. The combined soluble extracts were concentrated to dryness under reduced pressure in a rotary evaporator at $70 \pm 1^{\circ} \mathrm{C}$. The resulting crude aqueous extract was freeze dried, finally giving a dark brown, powdery aqueous extract residue. Without any further purification, aliquot portions of the aqueous extract residue were weighed and dissolved in distilled water (at room temperature) for use on each day of our experiments.

\section{Animals}

Sixty-two adult male Sprague-Dawley rats aged 9-10 weeks old ( $188.98 \pm 4.5 \mathrm{~g}$ ) were used for this study. The animals were bred and maintained at the Animal House of the Biomedical Resources Unit, University of KwaZulu-Natal. The animals received humane care in accordance with the Principles of Laboratory Animal Care of the National Medical Research Council and the Guide for the Care and Use of Laboratory Animals of the National Academy of Sciences (National Institute of Health Guide, 1985). The study protocol was approved by the University of KwaZulu-Natal Animal Ethics Committee (Ethical clearance number: 056/15/ Animals). All the rats were housed in well-ventilated plastic cages having dimensions of $36 \mathrm{~cm}$ long $\times 24 \mathrm{~cm}$ wide and $15 \mathrm{~cm}$ high. They were maintained under standardized animal house conditions (temperature: $28-31^{\circ} \mathrm{C}$; light: approximately $12 \mathrm{~h}$ natural light per day) and were fed with standard rat pellets from Meadow Feeds (a Division of Astral Operations Limited, Durban, South Africa) and given tap water ad libitum.

\section{Experimental design}

Two experimental (normoglycemic and glycemic) groups were considered healthy and diabetic rats. The normoglycemic control group $(n=6)$ comprised six animals while diabetic animals $(n=56)$ were divided into seven treatment groups: $\mathrm{B}-\mathrm{H}$ with eight animals per group and treated as follows:

- group A received water as a placebo (positive control);

- group B served as a diabetic control (negative control);

- group C received HAART cocktail (zidovudine, lamivudine and nevirapine) using the human therapeutic dose equivalents (600 mg, $300 \mathrm{mg}$ and $400 \mathrm{mg} /$ day respectively), as adjusted for animal weight to obtain corresponding therapeutic doses for the rat model [20];

- group D received $\mathrm{HH}$ extract (100 $\mathrm{mg} / \mathrm{kg}$ bwt);

- group E received $\mathrm{HH}$ extract (200 mg/kg bwt);

- group F received combined HAART cocktail and melatonin ( $5 \mathrm{mg} / \mathrm{kg}$ bwt);

- group G received combined HAART cocktail and $\mathrm{HH}$ extract (100 $\mathrm{mg} / \mathrm{kg}$ bwt) and

- group $\mathrm{H}$ received combined HAART cocktail and $\mathrm{HH}$ extract $(200 \mathrm{mg} / \mathrm{kg}$ bwt).

All administration was done daily by oro-gastric gavage and the treatments lasted for 8 weeks.

\section{Induction of type 2 diabetes mellitus (T2D)}

To generate the type 2 diabetes mellitus animal model, the diabetic groups were injected with a single intraperitoneal dose of $110 \mathrm{mg} / \mathrm{kg}$ body weight nicotinamide (Sigma, Saint Louis, MO, USA) in physiological saline, and 15 min later they were injected with $45 \mathrm{mg} / \mathrm{kg}$ body weight of streptozotocin (Sigma-Aldrich Chemical Com- 
pany, Missouri, St Louis, USA) dissolved in citrate buffer ( $\mathrm{pH} 4.5$ ) before intraperitoneal injection [21]. The control groups received vehicle citrate buffer through the same route. Seven days later, blood glucose concentrations of STZ-induced diabetic rats greater than $20 \mathrm{mmol} / /$ were considered diabetic.

Blood glucose, food and fluid intake, and weight changes

A One Touch Ultra Mini Glucometer (Contour TS, Switzerland) was used to estimate the blood glucose of treated and control animals. Blood obtained from the dorsal vein of the animals was used for estimation of capillary blood glucose concentrations on days $0,7,14,21,28,35,42,49$ and 56 (data not shown). Food and fluid intake was monitored on a daily basis during the experimental period (data not shown). Body weight (BW) changes of the animals were also recorded weekly. Body weight of the rats was recorded on the first day before the commencement of the treatment (the initial body weight), thereafter weekly and on the last day shortly before animal sacrifice (final body weight). Liver weights (LW) were measured by an electronic balance (Mettle Toledo; Microsep (Pty) Ltd, Greifensee, Switzerland) after laparotomy. Values are expressed as grams for all weight measurements.

\section{Tissue sample harvesting}

Eight weeks after treatment, all animals were sacrificed by exposure to halothane for $3 \mathrm{~min}$ via a gas anesthetic chamber $(100 \mathrm{mg} / \mathrm{kg})$ at the end of the experimental period. Thereafter, blood samples were collected by cardiac puncture into pre-cooled heparinized tubes and serum bottles, placed on ice for $3 \mathrm{~h}$ and centrifuged in a desktop centrifuge model 90-1 (Jiangsu Zhangji Instruments Co., China) for 15 min at 3000 revolutions per minute. The serum was decanted into Eppendorf tubes and stored at $-80^{\circ} \mathrm{C}$ for subsequent analysis. Liver tissues were excised, weighed and immediately fixed in 10\% neutral buffered formalin. After proper fixation, tissues were dehydrated in a graded series of alcohol, cleared in xylene and embedded in paraffin wax using a cassette.

\section{Determination of liver-function enzymes and total protein}

Biochemical analyses of the serum enzymes for ALT, AST, alkaline phosphatase (ALP) and total bilirubin (TB) were determined colorimetrically at 546 $\mathrm{nm}$ using Randox assay kits based on the principle first described by Reitman and Frankel [22]. Total protein was also measured using the calorimetric
Biuret method. In alkaline solution, peptide bonds bind with $\mathrm{Cu}^{2+}$ ions to form a blue violet colored complex. This complex is formed between the $\mathrm{Cu}^{2+}$ ion, the carbonyl oxygen and amide hydrogen atoms. Each $\mathrm{Cu}^{2+}$ ion is combined with six peptide bonds. The intensity of the color is proportional to the reacting number of peptide bonds, and therefore to the amount of protein present in the medium, in which the absorbance is measured at $546 \mathrm{~nm}$ [23].

\section{Determination of plasma glucose}

Glycemia was measured by the hexokinase method (Glucoquant; Boehringer-Mannheim) modified for a COBAS Bioautoanalyzer (Roche Biomedical, Basel, Switzerland).

\section{Determination of serum lipids}

Low- and high-density lipoproteins

Low-density lipoprotein (LDL) and high-density lipoprotein $(\mathrm{HDL})$ were determined by enzymatic methods according to Diniz et al. [24] using commercial diagnostic kits (Randox, UK).

\section{Triglyceride}

Triglyceride (TG) level is estimated by the enzymatic colorimetric method [25]. Triglyceride is measured using a series of coupled reactions in which triglycerides are hydrolyzed to produce glycerol and free fatty acids by the enzyme lipase. The glycerol is phosphorylated to glycerol-3-phosphate by glycerol kinase. The glycerol-3-phosphate is oxidized by glycerol phosphate oxidase, producing dihydroxyacetone phosphate and hydrogen peroxide. Peroxidase then catalyses the redox-coupled reactions of $\mathrm{H}_{2} \mathrm{O}_{2}$ with 4-aminoantipyrine (4-AAP), producing a bright purple color. Absorbance was measured at $540 \mathrm{~nm}$.

\section{Total cholesterol}

Cholesterol ( $\mathrm{CHOL})$ is determined by the enzymatic colorimetric method [26]. Cholesterol esters are hydrolyzed to free cholesterol by cholesterol ester hydrolase. The free cholesterol produced is oxidized by cholesterol oxidase to cholest-4-ene3-one with the simultaneous production of hydrogen peroxide, which oxidatively couples with 4-aminophenazone and phenol in the presence of peroxidase to yield a chromogen. The absorbance was measured at $400 \mathrm{~nm}$.

\section{Estimation of atherosclerotic and cardioprotective indexes}

The atherosclerotic index ( $\mathrm{Al})$ is a marker of atherosclerosis that has a direct correlation with 
the risk of cardiovascular disease. It is calculated by the formula [27]: $\mathrm{Al}=(\mathrm{TC}-\mathrm{HDL}) / \mathrm{HDL}$.

The cardioprotective index $(\mathrm{Cl})$ is a superior measure of the risk of cardiovascular disease. It was calculated as the ratio of HDL cholesterol to total cholesterol.

\section{Histopathological examination of liver tissues}

For routine histological study, the paraffin-embedded liver tissues were sectioned at $5 \mu \mathrm{m}$ thickness using a Leica RM 2255 microtome. The slides were deparaffinized in xylene and rehydrated in graded ethanol (100\%, 90\%, 80\%, 70\% and 50\%) and rinsed in water. Slides were stained in hematoxylin for $5 \mathrm{~min}$ and rinsed with water, and counterstained in eosin for general assessment of the liver structure. The tissues were also stained with Periodic acid Schiff' (PAS) for glycogen, neutral polysaccharides and basement membrane [28]. The stained slides were then cover slipped using DPX mounting glue directly over the tissue sections. Thereafter, the slides were left overnight to dry for examination under a light microscope. The sections were examined using a binocular microscope, Nikon Eclipse 80i, Tokyo, Japan (used to acquire the images). An independent histopathologist blinded to the treatment groups reported on the qualitative assessments of the slides.

\section{Statistical analysis}

All data were expressed as mean + standard deviation (SD). Statistical comparison of the differences between the control and experimental groups was performed with GraphPad InStat software (version 6.00, GraphPad Software, San Diego, California, USA), using one-way analysis of variance (ANOVA), by the Turkey-Kramer multiple comparison test. A value of $p<0.05$ was considered significant.

\section{Results}

\section{Mortality}

One animal died during the experimental period.

\section{Food and fluid intake and weight changes}

Food intake was not significantly different, whereas a significantly higher fluid intake was observed in all the diabetic groups compared to the control group (data not shown). The final body weights of rats in all groups were higher than the initial weight but the body weight difference and percentage gain were maximal in normoglycemic group A, recording a $66.73 \%$ increase (Table I). Upon injection of nicotinamide-STZ, there was a significant $(p<0.05)$ reduction in the body weight of diabetic animals with the least weight gain observed in the diabetic animals (groups $\mathrm{G}$ and $\mathrm{H}$ ) treated with HAART + HH $100 \mathrm{mg} / \mathrm{kg}$ and $200 \mathrm{mg}$ respectively (Table I). Similarly, there was no significant difference in the liver tissue weight of the control group A compared with diabetic control and groups $C$ and D treated with HAART and $H H(100 \mathrm{mg})$ alone $(p<0.05)$ respectively, but there was a significant difference $(p<0.001)$ in the liver tissue weight of the diabetic animals treated with $\mathrm{HH}(200 \mathrm{mg})$, and animals treated concomitantly with melatonin and $\mathrm{HH}(100 \mathrm{mg}$ and $200 \mathrm{mg}$ ) groups (E, F, G, and $H$ respectively) when compared with diabetic control group $B$. Also, the liver-body weight ratio was significantly different $(p<0.05)$ between normoglycemic control group $\mathrm{A}$ and the diabetic control, diabetic + HAART and diabetic + HAART + HH 200 mg treated groups.

\section{Biochemical parameters}

Tables II and III illustrate levels of blood biochemical tests in the nicotinamide-STZ diabetic

Table I. Body weight change

\begin{tabular}{|lccccccc|}
\hline Group & Treatment & BWi [g] & BWf $[\mathrm{g}]$ & BW diff $[\mathrm{g}]$ & \% BW diff & LW [g] & LBWR \\
\hline A & Control & $173.83 \pm 9.20$ & $289.83 \pm 23.41$ & 116.00 & 66.73 & $10.08 \pm 1.5$ & $3.47 \pm 0.3$ \\
\hline B & D-control & $209.13 \pm 14.17$ & $270.63 \pm 60.76$ & 61.50 & 29.41 & $11.48 \pm 2.7$ & $4.24 \pm 0.3^{\alpha}$ \\
\hline C & HAART-d & $213.25 \pm 14.25$ & $268.25 \pm 40.91$ & 55.00 & 25.79 & $11.23 \pm 1.8$ & $4.19 \pm 0.3^{\alpha}$ \\
\hline D & Hyp $_{100}$ & $196.25 \pm 18.61$ & $280.50 \pm 53.70$ & 84.25 & 42.93 & $10.95 \pm 1.4$ & $3.98 \pm 0.5$ \\
\hline E & Hyp $_{200}$ & $187.63 \pm 20.68$ & $242.38 \pm 50.20$ & 54.75 & 29.18 & $9.11 \pm 1.2^{\beta}$ & $3.81 \pm 0.4$ \\
\hline F & HAART + Mel & $181.13 \pm 19.75$ & $231.25 \pm 59.05$ & 50.12 & 27.67 & $8.27 \pm 1.6^{\beta}$ & $3.65 \pm 0.5^{\beta}$ \\
\hline G & HAART + Hyp & $186.88 \pm 56.42$ & $214.25 \pm 26.44$ & 27.37 & $14.65^{\alpha}$ & $8.49 \pm 1.3^{\beta}$ & $3.96 \pm 0.3$ \\
\hline H & HAART + Hyp $_{200}$ & $186.75 \pm 17.47$ & $213.00 \pm 52.10$ & 26.25 & $14.06^{\alpha}$ & $8.98 \pm 1.7^{\beta}$ & $4.26 \pm 0.3^{\alpha}$ \\
\hline
\end{tabular}

Data are shown as mean $\pm S D ; p<0.05^{\alpha \beta}$ vs. group $A^{\alpha}$ and $p<0.001$ group $B^{\beta}$ (Turkey-Kramer multiple range post-hoc test). BWi-initial body weight, $B W f$ - final body weight, $B W$ - body weight, $L W$ - liver weight, $L B W R$ - liver-body weight ratio. 
Table II. Plasma glucose and liver function parameters

\begin{tabular}{|lcccccc|}
\hline Group & $\begin{array}{c}\text { Glucose } \\
{[\mathrm{mmol} / \mathrm{l}]}\end{array}$ & AST [IU/I] & ALT [IU/I] & AST/ALT ratio & ALP [IU/I] & TB [IU/I] \\
\hline A & $5.57 \pm 0.17$ & $150.00 \pm 9.12$ & $72.67 \pm 9.81$ & $2.11 \pm 0.53$ & $224.70 \pm 20.21$ & $3.00 \pm 0.00$ \\
\hline B & $44.87 \pm 4.47^{\alpha}$ & $134.30 \pm 5.35$ & $136.70 \pm 24.10^{\alpha}$ & $1.13 \pm 0.40^{\alpha}$ & $1044.00 \pm 35.19^{\alpha}$ & $4.33 \pm 2.73$ \\
\hline C & $66.47 \pm 2.54^{\alpha}$ & $124.00 \pm 3.40$ & $174.70 \pm 23.94^{\alpha}$ & $0.72 \pm 0.08^{\alpha}$ & $2062.00 \pm 13.67^{\alpha \beta}$ & $6.00 \pm 0.89^{\alpha \beta}$ \\
\hline D & $37.85 \pm 0.93^{\alpha}$ & $140.00 \pm 9.36$ & $134.70 \pm 14.92^{\alpha}$ & $1.09 \pm 0.23^{\alpha}$ & $1711.00 \pm 13.28^{\alpha \beta}$ & $6.00 \pm 1.10^{\alpha \beta}$ \\
\hline E & $36.00 \pm 3.70^{\alpha}$ & $139.30 \pm 1.12$ & $120.00 \pm 12.10^{\alpha}$ & $1.17 \pm 0.13^{\alpha}$ & $1060.00 \pm 33.40^{\alpha}$ & $6.33 \pm 2.25^{\alpha \beta}$ \\
\hline F & $50.60 \pm 2.17^{\alpha}$ & $132.70 \pm 4.96$ & $127.30 \pm 13.19^{\alpha}$ & $1.05 \pm 0.09^{\alpha}$ & $1125.00 \pm 20.71^{\alpha}$ & $3.33 \pm 1.86$ \\
\hline G & $36.27 \pm 0.88^{\alpha}$ & $108.70 \pm 6.75^{\alpha}$ & $145.30 \pm 19.81^{\alpha}$ & $0.76 \pm 0.12^{\alpha}$ & $1761.00 \pm 37.07^{\alpha \beta}$ & $6.00 \pm 0.00^{\alpha \beta}$ \\
\hline H & $50.40 \pm 5.75^{\alpha}$ & $143.70 \pm 7.83$ & $184.70 \pm 9.97^{\alpha}$ & $0.78 \pm 0.18^{\alpha}$ & $1291.00 \pm 29.75^{\alpha}$ & $6.33 \pm 2.25^{\alpha \beta}$ \\
\hline
\end{tabular}

Data are shown as mean $\pm S D ; p<0.05 / 0.001 / 0.0001^{\alpha \beta}$ vs. group $A^{\alpha}$ and group $B^{\beta}$ (Turkey-Kramer multiple range post-hoc test). AST - aspartate aminotransferase, ALT - alanine aminotransferase, ALP - alkaline phosphatase, TB - total bilirubin.

Table III. Total protein and lipid profiles

\begin{tabular}{|lccccccc|}
\hline Group & $\begin{array}{c}\text { Total protein } \\
{[\mathrm{g} / \mathrm{l}]}\end{array}$ & $\mathrm{LDL}[\mathrm{mmol} / \mathrm{l}]$ & $\begin{array}{c}\mathrm{HDL} \\
{[\mathrm{mmol} / \mathrm{l}]}\end{array}$ & $\begin{array}{c}\text { Total } \\
\text { cholesterol } \\
{[\mathrm{mmol} / \mathrm{l}]}\end{array}$ & $\begin{array}{c}\text { Triglycerides } \\
{[\mathrm{mmol} / \mathrm{l}]}\end{array}$ & $\mathrm{Al}$ & $\mathrm{Cl}$ \\
\hline $\mathrm{A}$ & $65.67 \pm 1.4$ & $-0.04 \pm 0.2$ & $0.72 \pm 0.0$ & $1.03 \pm 1.0$ & $0.76 \pm 0.5$ & $0.43 \pm 0.1$ & $0.72 \pm 0.0$ \\
\hline B & $59.67 \pm 0.5$ & $-0.52 \pm 0.3^{\alpha}$ & $1.05 \pm 0.2$ & $1.47 \pm 0.2^{\alpha}$ & $2.03 \pm 0.7^{\alpha}$ & $0.40 \pm 0.1$ & $0.72 \pm 0.0$ \\
\hline C & $61.33 \pm 1.9$ & $-0.45 \pm 0.3$ & $1.03 \pm 0.0$ & $1.30 \pm 0.2$ & $1.58 \pm 0.3$ & $0.27 \pm 0.2$ & $0.80 \pm 0.1$ \\
\hline D & $58.00 \pm 3.3^{\alpha}$ & $-0.30 \pm 0.2$ & $1.04 \pm 0.0$ & $1.57 \pm 0.2^{\alpha}$ & $1.79 \pm 0.7^{\alpha}$ & $0.50 \pm 0.2$ & $0.68 \pm 0.1$ \\
\hline E & $60.67 \pm 2.3$ & $-0.24 \pm 0.1$ & $1.08 \pm 0.2$ & $1.80 \pm 0.2^{\alpha}$ & $2.09 \pm 0.3^{\alpha}$ & $0.69 \pm 0.1$ & $0.59 \pm 0.0$ \\
\hline F & $62.67 \pm 1.9$ & $-0.23 \pm 0.3$ & $1.17 \pm 0.1$ & $1.57 \pm 0.3^{\alpha}$ & $1.37 \pm 0.6$ & $0.33 \pm 0.2$ & $0.76 \pm 0.1$ \\
\hline G & $63.00 \pm 0.9$ & $-0.45 \pm 0.2$ & $1.10 \pm 0.1$ & $1.50 \pm 0.2^{\alpha}$ & $1.83 \pm 0.3^{\alpha}$ & $0.36 \pm 0.1$ & $0.74 \pm 0.0$ \\
\hline H & $58.67 \pm 6.9$ & $-1.17 \pm 0.3^{\alpha \beta}$ & $1.07 \pm 0.1$ & $1.47 \pm 0.1^{\alpha}$ & $3.38 \pm 0.9^{\alpha \beta}$ & $0.38 \pm 0.1$ & $0.73 \pm 0.1$ \\
\hline
\end{tabular}

Data are shown as the mean $\pm S D ; p<0.05 / 0.001 / 0.0001^{\alpha \beta}$ vs. group $A^{\alpha}$ and group $B^{\beta}$ (Turkey-Kramer multiple range post-hoc test). $L D L$ - low-density lipoprotein, $H D L$ - high-density lipoprotein, $A l$-atherosclerotic index, $\mathrm{Cl}$-cardioprotective index.

and normal rats following the 8-weeks experimental period.

Plasma glucose, serum AST, ALT, ALP and total bilirubin in the experimental study

The plasma glucose levels remained elevated in all the diabetic animals treated with $\mathrm{HH}$, HAART and combination of the $H H$ and HAART throughout the period of treatment (Table II). There were changes in the functional hepatotoxicity indices such as AST, ALT ALP and total bilirubin (Table II). There was observed a decrease in the AST levels in all the diabetic animals (groups B-H) except in group G (HAART + HH $100 \mathrm{mg}$ ) animals, in which it was statistically difference $(p<0.001)$ as compared with the normoglycemic control group $A$. Also, there were statistically significant higher $(p<0.05)$ ALT levels of all the diabetic (groups $\mathrm{B}-\mathrm{H})$ animals treated with HAART alone and/or concomitantly with $\mathrm{HH}$ (100 and $200 \mathrm{mg}$ ) com- pared with the normoglycemic control group A. The AST/ALT ratio follows the same pattern of ALT as shown in Table II. However, when compared with the diabetic control group $B$, the levels of AST and ALT were not significantly $(p<0.001)$ different in diabetic treated animals. Similarly, the levels of ALP in all the diabetic (groups B-H) animals treated with HAART alone and/or concomitantly with $\mathrm{HH}$ (100 and $200 \mathrm{mg}$ ) were statistically $(p<0.05)$ elevated compared with the normoglycemic control group A. However, when compared to the diabetic control group B, the levels of ALP were also significantly elevated in the diabetic groups treated with HAART, HH $100 \mathrm{mg}$ and HAART + HH $100 \mathrm{mg}$. In addition, aside from the diabetic animals treated with HAART + melatonin (group F), there were statistically significant difference in the levels of total bilirubin in diabetic (groups C, $D, E, G$ and $H$ ) animals relative to animals in normoglycemic and diabetic control groups $(p<0.05 /$ $p<0.0001$ respectively), as shown in Table II. 
Serum total protein and lipid profiles following treatment with HAART, melatonin and $H H$

Table III shows the data for serum total protein and lipid profile in different animal groups. There was no significant difference in the level of total protein in the treated and non-treated diabetic (groups B-H) animals compared with the normoglycemic control group A, except for the animals treated with $H H 100 \mathrm{mg}$. However, when compared with diabetic control animals in group B, the levels of total protein in groups $\mathrm{C}-\mathrm{H}$ animals treated with HAART alone and/or concomitantly with $H H$ (100 and $200 \mathrm{mg}$ ) or melatonin were not significantly different $(p<0.0001)$. There were significant $(p<0.001)$ changes in the levels of total serum cholesterol of all treated and non-treated diabetic animals (groups B, D-H), with the exception of group $C$ animals that were administered with HAART alone, in which there was not a significant difference compared with the normal control.

In addition, serum triglycerides were significantly $(p<0.05)$ elevated in diabetic control (group B) and all diabetic treated animals (groups $\mathrm{C}-\mathrm{H}$ ) compared with the normoglycemic animals. There was also difference in the level of serum $\mathrm{HDL}$ of animals in groups $\mathrm{C}-\mathrm{H}$ animals treated with HAART alone and/or concomitantly with HH (100 and $200 \mathrm{mg}$ ) or melatonin; however, the changes in HDL was not statistically different when compared to the normal and diabetic control groups A and B respectively $(p<0.0001)$. There was also a statistically significant difference in the serum levels of $L D L$ in groups $B$ and $H$ $(p<0.001)$, whereas diabetic groups $(C-G)$ treated with HAART alone, HH 100 and $200 \mathrm{mg}$ alone, HAART + melatonin as well as those that received HAART + HH $100 \mathrm{mg}$ all non-significant changes in the parameter compared with the normoglycemic control group A $(p<0.0001)$. In addition, there was no significant difference in the atherosclerotic and cardioprotective indexes of all treated and non-treated diabetic groups B, D-H $(p<0.001)$ (Table III).

\section{Histopathological changes in liver tissue (H\&E and PAS)}

The H\&E stained sections of the liver tissues in control animals revealed adequate preservation of hepatocellular architecture with the central vein (with blood) and cords of hepatocytes arranged in peripherally radiating fashion with accumulation of golden brown finely granular lipofuscin pigments and associated sinusoid spaces with normal fatty vacuolation of liver cells (Figure $1 \mathrm{~A}$ ). The outline of hepatocytes and sinusoidal spaces were seen with no pathologies. Histopathological evaluation of all other groups showed various degrees of distortions. The liver tissue sections of all diabetic animals revealed mild to severe distortion (Figures $1 \mathrm{~B}-\mathrm{H}$ ). The diabetic animals treated with HAART alone, HAART + melatonin as well as those treated with HAART + HH (100 and $200 \mathrm{mg} / \mathrm{kg}$ ) adjuvant showed varying cytoarchitectural patterns with slight to severe distortions including extensive necrosis of hepatocytes with enlarged sinusoidal appearance and severe loss of polyhedral arrangement of cords of cells. Where hepatic sinusoids had become occluded, central veins were congested and hepatocytes appeared swollen; however, the disruption was more pronounced in the diabetic animals treated with $100 \mathrm{mg} / \mathrm{kg}$ alone with severe necrosis, isolated hepatocytes without prominent nucleoli and loss of polyhedral arrangement of cords of cells and enlarged sinusoidal appearance (Figure 1 D).

Photomicrographs of the liver tissue sections stained with PAS (Figure 2 A) showed presence of glycogen in the tissues, depicted by pink color in the sections. The normoglycemic liver tissue sections revealed normal liver architecture, containing a large amount of glycogen with bright pink hepatocytes, neutral polysaccharides and basement membranes. However, the liver tissue sections from diabetic groups $\mathrm{G}, \mathrm{D}, \mathrm{B}, \mathrm{C}, \mathrm{F}, \mathrm{H}$ and $E$ showed reduction in PAS-staining intensity. The extensive and generalized sections of diabetic animals in groups D and G treated with $H H 100 \mathrm{mg}$ alone and HAART + HH $100 \mathrm{mg} / \mathrm{kg}$ respectively revealed a large amount of glycogen in the hepatocytes as well as cords/sinusoidal spaces with bright pink hepatocytes, neutral polysaccharides and basement membranes that were PAS-positive. As PAS stain demonstrated presence of glycogen, indications for various degrees of distortions in hepatocellular cords were also evident in the grading of the intensities (Figure $2 \mathrm{~A}-\mathrm{H}$ ).

\section{Discussion}

As people living with HIV/AIDS continue to benefit from the reduction in morbidity and mortality due to HAART, organ toxicities are frequently becoming a major concern in view of associated consequences that may predispose to metabolic complications. The liver performs numerous vital metabolic activities, including biochemical, synthetic and excretory functions. Consequently, key roles executed by the liver in the clearance and transformation of chemicals expose the organ to toxic injury [29]. Our current study revealed that diabetes coupled with the administration of HAART and Hypoxis hemerocallidea $(H H)$ concomitantly leads to changes in the liver function markers, proteins, and lipids and a reduction in quali- 

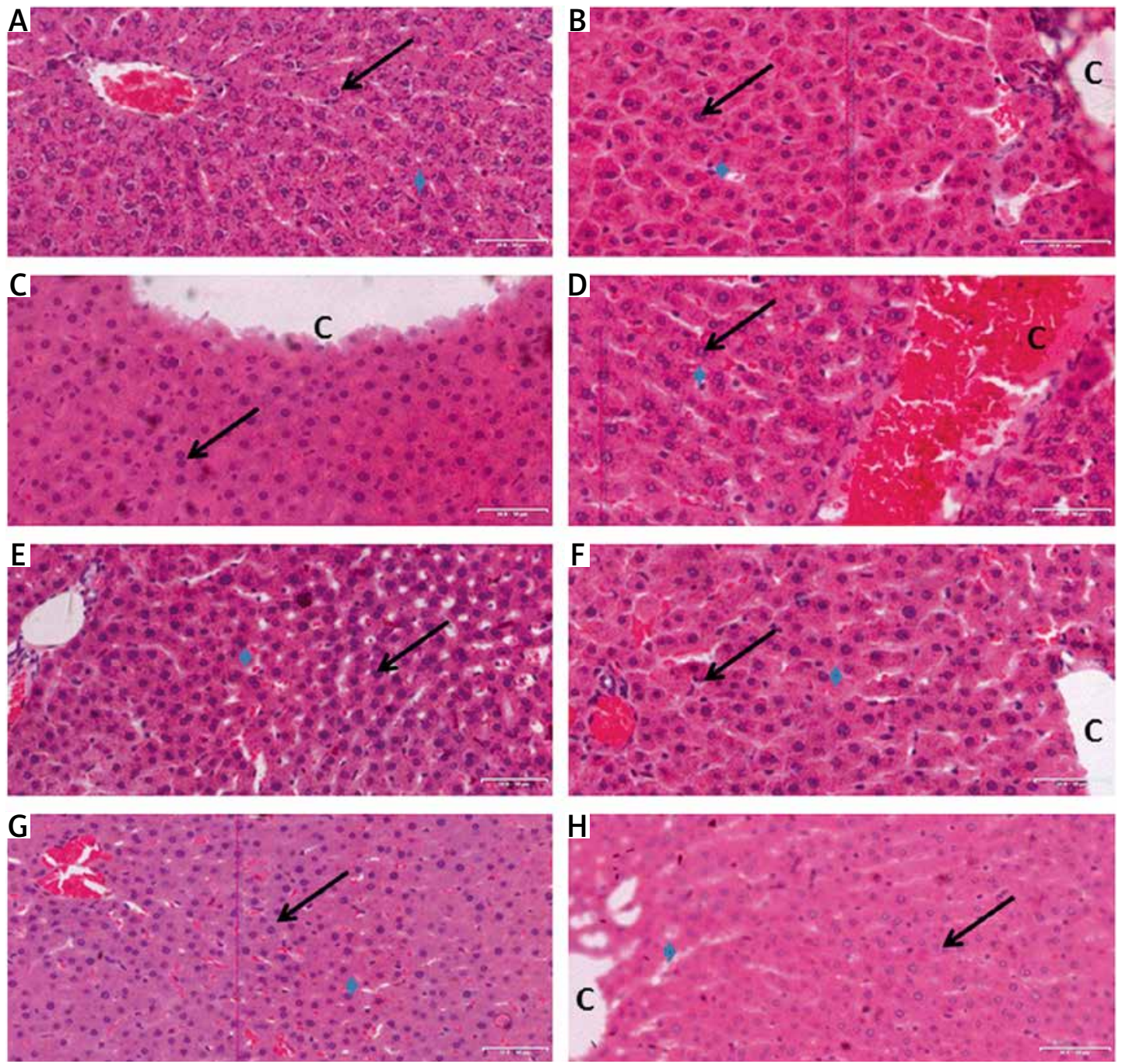

Figure $1 \mathrm{~A}-\mathrm{H}$. Representative photomicrographs of the liver sections in rats of: A - negative control, the morphology of the liver is normal as indicated by intact central vein (C), sinusoids (arrowhead), and hepatocytes (arrows) (B): DBT (Diabetic) control, sinusoids have become largely occluded, perhaps due to swollenn hepatocytes (arrows); central vein (C) is also congested, C - DBT + HAART, D - DBT + $100 \mathrm{mg} / \mathrm{kg} \mathrm{HH,} \mathrm{E-DBT} \mathrm{+} 200 \mathrm{mg} / \mathrm{kg} \mathrm{HH,} \mathrm{F-DBT} \mathrm{+}$ HAART + melatonin, G - DBT + HAART + $100 \mathrm{mg} / \mathrm{kg} H H, \mathrm{H}-\mathrm{DBT}+\mathrm{HAART}+200 \mathrm{mg} / \mathrm{kg} H H$ (H \& E stain)

tative evaluated glycogen in our protocol. This in addition to its inability to mitigate hepatocellular morphological changes further supports its herbdrug interaction (HDI) effects. It is possible that unfavorable HDI between $H H$ and HAART could affect the efficacy or safety of the latter.

Diabetes mellitus is characterized by absolute or relative deficiencies in insulin secretion and/or insulin action associated with chronic hyperglycemia and disturbances of carbohydrate, lipid and protein metabolism. Long-term vascular complications represent a major cause of morbidity and mortality in patients with diabetes mellitus. In addition, various biochemical disorders are associated with vascular complications, such as hyperlipidemia and oxidative stress, which frequently co-exist with diabetes mellitus [30]. The current study revealed that antiretroviral therapy (HAART) with $H H$ induced negative changes in the body weight of experimental animals especially in the diabetic + HAART + HH (100 and $200 \mathrm{mg} / \mathrm{kg})$ animals. While loss of weight may be associated with induction of diabetes, initiation of HAART may also contribute to the low pace of weight gain due to other metabolic activities relative to lipid and sugar [31]. While there was an increase in the organ weight of diabetic control and HAART-treated diabetic animals, and there was a reduction in the liver weights of diabetic animals treated with HAART + melatonin, HAART + HH of both dosages relative to diabetic control animals, this reduction of liver weight may result from hepatic atrophy in diabetic rats. As earlier reported, end organ toxicity can manifest in the form of organ swelling, atrophy or hypertrophy [32]. The liver-body weight ratio of the diabetic experimental animals was also significantly affected, especially in diabetic animals treated with $200 \mathrm{mg}$ of $\mathrm{HH}$ concomitantly 

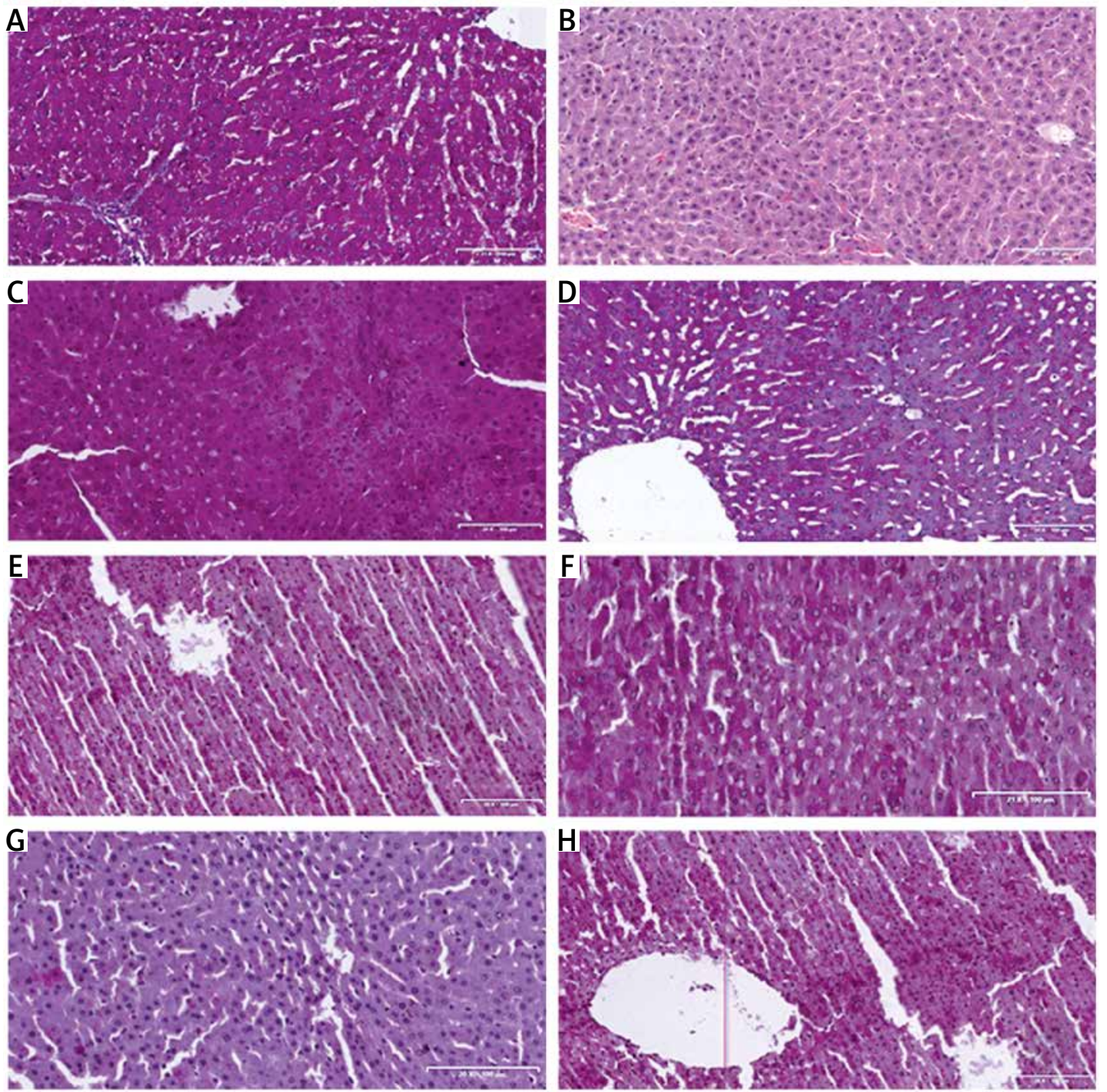

Figure 2 A-H. Representative photomicrographs of the liver sections in rats of: A - negative control, B - DBT (diabetic) control, sinusoids have become largely occluded, perhaps due to swollen hepatocytes; central vein $(C)$ is also congested, C - DBT + HAART, D - DBT + $100 \mathrm{mg} / \mathrm{kg} \mathrm{HH,} \mathrm{E} \mathrm{-} \mathrm{DBT} \mathrm{+} 200 \mathrm{mg} / \mathrm{kg} \mathrm{HH,} \mathrm{F} \mathrm{-} \mathrm{DBT} \mathrm{+} \mathrm{HAART} \mathrm{+} \mathrm{melatonin,}$ $\mathrm{G}-\mathrm{DBT}+\mathrm{HAART}+100 \mathrm{mg} / \mathrm{kg} H H, \mathrm{H}-\mathrm{DBT}+\mathrm{HAART}+200 \mathrm{mg} / \mathrm{kg} H H$ (PAS Stain)

with HAART, which were seen to be more negatively affected in this parameter. The weight coefficient (organ-body weight ratio) is a sensitive indicator of the toxicology and therefore important in identification of target organ [18].

The aminotransferases are the most frequently utilized and specific indicators of hepatocellular necrosis. These enzymes - AST and ALT - catalyze the transfer of the amino acids of aspartate and alanine respectively to the ketoglutaric acid, which are indicators in understanding necrotic and inflammatory changes in the liver [33]. In our current investigation, ALT levels of all the diabetic animals treated with HAART, $H H$ (both doses), HAART + melatonin and HAART + HH (both doses) concomitantly were found to be significantly elevated relative to the normoglycemic control animals, whereas the AST levels were down regulated in the same groups of animals. The hepatic causes of increased serum ALT activity, with or without increased AST activity, include hepatocellular necrosis, injury, or regenerative/reparative activity [34], in which the different levels of these enzymes may be associated with either diabetic complications and/or herbal-drug interaction (HDI). In search for better outcomes and wellbeing of individuals living with HIV/AIDS, the use of alternative medicines to mitigate the metabolic complications is required as the use of HAART has been implicated in HAART-induced liver injury [18]. $\mathrm{HH}$ at both dosages concomitantly with HAART in diabetes showed appreciable possibilities to cause an increase in liver enzymes relative to the HAART regimen in the normoglycemic situation. Primarily, ALT is localized to the liver but AST is present in a wide variety of tissues such as the heart, skele- 
tal muscle, kidney, brain and liver [35]. Therefore, the activity of ALT and AST in the serum at any moment reflects the relative rate at which they enter and leave the circulation. The elevation of ALT is probably due to abundant pyridoxine. The ratio of AST to ALT is of use in drug-induced toxicity and alcoholic liver disease. Mild elevations of these enzymes are usually seen in drug toxicity, extrahepatic biliary atresia (EHBA), fatty liver, cirrhosis, and non-alcoholic steatohepatitis (NASH) [35]. In viral hepatitis, the ratio is usually less than one. The ratio invariably rises to more than one as cirrhosis develops, possibly because of reduced plasma clearance of AST secondary to impaired function of sinusoidal cells. Co-infections with other hepatocellular viral infections such as hepatitis B virus and hepatitis C virus may further be responsible to liver-related severe morbidity and mortality [36].

In addition, the elevation of serum ALP levels in all diabetic animals treated and non-treated was more than five-fold. ALP is an indicator of hepatobiliary injury most found in the circulation and originates from liver or bone [34]. As noted in our current study, the ALP levels were seven to tenfold elevated in the diabetic animals treated with HAART, HH (100 mg) and HAART + HH (100 mg). This exacerbated level of ALP points towards the implication of organ injury associated with HDI in diabetic comorbidity in the HAART regimen. Moreover, the total bilirubin (TB) level was also found to be elevated in all diabetic animals. Hyperbilirubinemia seen in HDI and acute viral hepatitis is directly proportional to the degree of histological injury of hepatocytes and the longer course of the disease [35]. The unconjugated (indirect-reacting) fraction of bilirubin generated from the degradation of erythrocytes and the conjugated (direct-reacting) fraction together comprise the TB concentration. The TB levels were noted to be double in diabetic animals treated with HAART alone, $\mathrm{HH}$ (both doses) and HAART + HH (both doses) compared with normoglycemic and untreated diabetic animals, suggesting further disruptions in the liver tissues. This is in addition to other liver function indices revealing the derangement in the liver tissue. Increases in the serum TB concentration are generally the result of bile retention subsequent to impairment of intrahepatic or extrahepatic bile flow (cholestasis), increased production associated with accelerated erythrocyte destruction, or altered bilirubin metabolism. Bilirubin fractionation is generally only of value for exploring drug-related inhibition of the bilirubin-conjugating enzyme [34].

The liver is the major source for most of the serum proteins. The parenchymal cells are responsible for synthesis of albumin, fibrinogen and other coagulation factors and most $a$ and $b$ globulins
[35]. In this study, there was observed a reduction in the levels of total protein of all the treated and untreated diabetic animals when compared with the normoglycemic animals except for the diabetic animals treated with $H H(100 \mathrm{mg})$. The serum protein levels tend to be normal in diseases such as acute viral hepatitis, drug-related hepatotoxicity and obstructive jaundice. As reported by Kumarappan et al., hypoalbuminemia is not specific for liver disease and may occur in protein malnutrition, nephritic syndrome and chronic protein losing enteropathies [37].

Assessment of lipid profile is of significant importance in evaluation of cardiovascular outcomes following use of HAART. Introduction of combination antiretroviral therapy is associated with the syndrome of subcutaneous lipoatrophy, central adiposity, dyslipidemia, and insulin resistance, termed HIV-associated lipodystrophy (HIVLD) [38]. The exposure was primarily associated with $\mathrm{Pl}$, but with exposure to nucleoside reverse transcriptase inhibitors (NRTIs) later on [39], particularly thymidine analogs (tNRTIs) such as stavudine and zidovudine [40] were also recognized as being central to the development of this syndrome. In the current study, there was a significant increase in levels of these lipids in all treated and untreated diabetic animals, though diabetes mellitus is usually associated with hyperlipidemia [41]. Furthermore, there were observational alterations in atherosclerotic and cardioprotective indexes. A high level of the atherosclerotic index and lower level of the cardioprotective index are usually found in diabetes and such an elevation represents a risk factor for coronary heart disease [42].

Moreover, morphological and physiological studies have identified the hepatic tissue as a site of attack by HAART, resulting in the derangement of the hepatocellular cords and sinusoidal spaces. Moreover, hepatocellular injury has been identified as a potential flaw with most antiretroviral regimens, especially with nevirapine and stavudine [43]. Therefore, the deviations in the hepatocellular architecture in HAART-treated animals as well as all diabetic-treated animals and the inability of $\mathrm{HH}$ adjuvant to mitigate the derangements resulting from hepatocellular distortions suggest that the latter has herb-drug interaction (HDI). Our result on co-administration of melatonin does not support the concurrent use of this antioxidant as a supplement by PLWHA. While there are numerous achievements in its use as discussed extensively by Guneli et al. [44], accumulating evidence from our laboratory [45] on the exacerbation of organ injuries has been published previously. It is believed that concurrent administration of melatonin alter bioavailability, metabolism and pharmacokinetics of certain HIV medications, rais- 
ing concerns about the possibility of HAART and melatonin supplementation leading to increased toxicity. Contrary to previous claims of the anti-inflammatory, antimicrobial, anti-diabetic, anticonvulsant and anticancer activities of $\mathrm{HH}$ reported by various authors [16, 17], at different dosages of $\mathrm{HH}$ in our protocol, the hypoglycemic effect was negative as the glycemia levels remained elevated throughout the course of treatment with $\mathrm{HH}$ in the current study.

We also observed an increase in the level of serum HDL of diabetic animals treated with HAART alone and/or concomitantly with HH (100 mg and $200 \mathrm{mg}$ ) or melatonin though not statistically different when compared to the normoglycemic and diabetic control animals. However, the serum levels of LDL in diabetic animals treated with HAART alone, $H H$ 100/200 mg/kg alone, HAART + melatonin as well as HAART + HH $100 \mathrm{mg}$ all showed an observational decline in the parameter compared with the normoglycemic control animals. However, the downregulation was not several-fold. These effects are suspected to be as a result of herbdrug interaction and diabetic-induced alterations. In addition to HIVLD, in the general population there is concern about the increasing prevalence of the metabolic syndrome among HIV-infected individuals. The abdominal obesity, low HDL-C, high triglycerides, and insulin resistance, all seen in HIVLD, are also components of the metabolic syndrome [46, 47]. An interesting finding which points towards a hepatic cause of dyslipidemia is the fact that hepatitis $\mathrm{C}$ virus (HCV) co-infection may appear to protect against the development of HAART-associated dyslipidemia. This may be due to alterations in hepatocyte lipid secretion-mono-infection with HCV associated with lower serum cholesterol but a marked increase in hepatic steatosis. The ultimate result of abnormally functioning subcutaneous adipose tissue is reduced storage capacity for circulating lipids resulting in increased circulating free fatty acids, reduced adiponectin secretion and lipid accumulation in non-adipose tissues such as liver (hepatic steatosis) and hepatic triglyceride accumulation, which was even elevated relative to the normoglycemic control animals in this current study. In correlations with PAS-staining intensities of the liver sections, there is supporting evidence. The administration of HAART and HH concomitantly leads to a reduction in qualitative evaluation of glycogen in this protocol. This in addition to its inability to mitigate hepatocellular morphological changes further supports HDI [18] and diabetic [41] effects resulting from its concomitant use with HAART. It is possible that unfavorable herb-drug interactions between $\mathrm{HH}$ and HAART could affect efficacy or safety of the latter. This finding is in line with Mills et al.
[48]; the interactions between extracts of $\mathrm{HH}$ and antiretroviral drugs inhibited CYP3A4, an isoform of cytochrome P450 and drug transporter protein (P-glycoprotein). In addition, $\mathrm{HH}$ extracts have been claimed to activate drug nuclear receptor pregnane $X(P X R)$, which modulates expressions of both CYP3A4 and p-glycoprotein [49]. Many antiretroviral drugs are substrates of CYP3A4 and some herbal preparations are known to alter blood levels of these drugs through their effects on CYP3A4 and p-glycoprotein. It is therefore reasonable to argue that $\mathrm{HH}$ could potentially interact with HIV drug metabolizing enzymes; thus patients taking $\mathrm{HH}$ extracts concurrently with antiretroviral drugs may be at risk of developing adverse metabolic complications which may lead to treatment failure, viral resistance and hepatocellular toxicity while the search for herbal adjuvants to mitigate comorbid metabolic complications continues.

In conclusion, from this study, metabolic perturbations and hepatic injuries resulting from HAART and induced-diabetes were not mitigated by $H H$ adjuvants, as observed in the metabolic and histopathological assessments. There is no positive outcome in concomitant use of melatonin with HAART either. Therefore, caution is needed following the over-the-counter use of $\mathrm{HH}$ extracts by PLWHA as an immune booster as the risks of exacerbation of hepatic injuries are high without promising hypoglycemic activity of the plant. However, more studies are required to further support these findings.

\section{Acknowledgments}

We acknowledge the award of operational funds by the College of Health Science, University of KwaZulu-Natal to postgraduate student Onanuga IO. Technical support from Dr. Sanil Singh and Dr. Linda Bester of the Biomedical Research Unit, University of KwaZulu-Natal is acknowledged. This work is based on research supported in part by the National Research Foundation (NRF) of South Africa for the grant, Unique Grant No. U99053 to the senior author.

\section{Conflict of interest}

The authors declare no conflict of interest.

\section{References}

1. Kushnir VA, Lewis W. Human immunodeficiency virus/ acquired immunodeficiency syndrome and infertility: emerging problems in the era of highly active antiretrovirals. Fertil Steril 2011; 96: 546-50.

2. Young F, Critchley JA, Johnstone LK, Nigel C, Unwin NC. A review of co-morbidity between infectious and chronic disease in Sub-Saharan Africa: TB and diabetes mellitus, HIV and metabolic syndrome, and the impact of globalization. Global Health 2009; 5: 9. 
3. Konstantinos M, Gesthimani M, Athina K, Basil CT, Dimitrios GG. The complex interaction between obesity, metabolic syndrome and reproductive axis: a narrative review. Metabol Clin Exp 2013; 62: 457-78.

4. Kalra S, Kalra B, Agralwal N, Unnikrishnam AG. Understanding diabetes in patients with HIV/AIDS. Diabeto Metab Syndr 2011; 3: 1-8.

5. Grinspoon S, Donovan D Jr, Bilezikian J. Aetiology and pathogenesis of hormonal and metabolic disorders in HIV infection. Baillere's Clin Endocrinol Metab 1994; 8: 735-55.

6. Grinspoon S, Carr A. Cardiovascular risk and body fat abnormalities in HIV-infected adults. N Engl J Med 2005; 352: 48-62.

7. De Wit S, Sabin CA, Weber R, et al. Incidence and risk factors for new-onset diabetes in HIV-infected patients. The Data Collection on Adverse Events of Anti-HIV Drugs (D:A:D) Study. Diabetes Care 2008; 31: 1224-29.

8. Abrescia N, Abbraccio MD, Figoni M, Busto A, Maddaloni A, De Marco M. Hepatotoxicity of antiretroviral drugs. Curr Pharm Des Rev 2005; 11: 3697-710.

9. Ramana KV, Ratna R. Abnormal levels of gamma-glutamyl transpeptidase (GGTP), ALT, AST in human immu nodeficiency virus-1 (HIV-1) infection. Biochem Physio 2012; 1: 101.

10. Robinson FP, Quinn LT, Rimmer JH. Effects of high-intensity endurance and resistance exercise on HIV metabolic abnormalities: a pilot study. Biol Res Nurs 2007; 8: 177-85.

11. Valiathan MS. Healing plants. Curr Sci 1998; 75: 112-6.

12. Nair VDP, Dairam A, Agbonon A, Arnason JT, Foster BC, Kanferj I. Investigation of the antioxidant activity of African potato (Hypoxis hemerocallidea). Agric Food Chem 2007; 55: 1707-11.

13. Laporta O, Perez-Fons L, Mallavia R, Caturla N, Micol V. Isolation, characterisation and antioxidant activity assessment of bioactive compounds derived from Hypoxis rooperi corm extract (African Potato). Food Chem 2007; 101: 1425-37.

14. Kruger PB, Albrecht CF, Liebenberg RW, Van Jaarsveld PP. Studies on hypoxoside and rooperol analogues from Hypoxis rooperi and Hypoxis latifolia and their biotransformation in man using high performance liquid chromatography with inline sorption enrichment and diode array detection. J Chromatogr 1994; 662: 71-8.

15. Moreau RA, Bruce DW, Kevin BH. Phytosterols, phytostanols, and their conjugates in foods: structural diversity quantitative analysis, and health-promoting uses. Prog Lipid Res 2002; 41: 457-500.

16. Ncube B, Finnie JF, van Staden J. Seasonal variation in antimicrobial and phytochemical properties of frequently used medicinal bulbous plants from South Africa. S Afr J Bot 2011; 77: 387-96.

17. Ojewole JAO, Kamadyaapa DR, Musabayane CT. Some in vitro and in vivo cardiovascular effects of Hypoxis hemerocallidea Fisch \& C.A. Mey (Hypoxidaceae) corm (African potato) aqueous extract in experimental animal models. Cardiovasc J South Afri 2006; 17: 166-71.

18. Azu OO, Jegede Al, Offor U, Onanuga IO, Kharwa S, Naidu ECS. Hepatic histomorphological and biochemical changes following highly active antiretroviral therapy in an experimental animal model: does Hypoxis hemerocalleadea exacerbate hepatic injury? Toxicol Rep 2016; 3: 114-22.

19. Ojewole JA, Awe EO, Nyinawumuntu A. Antidiarrheal activity of Hypoxis hemerocallidea Fisch. \& C. A. Mey. (Hypoxidaceae) corm ('African potato') aqueous extract in rodents. Phytother Res 2009; 23: 965-71.
20. Umar RA, Hassan SW, Ladan MJ, et al. Adverse effects associated with antiretroviral therapy drugs (nevirapuine, lamivudine and stavudine) to albino rats: implication for management of patients with HIV/AIDS. Asian J Biochem 2000; 3: 19-25.

21. Mojani MS, Sarmadi VH, Vellasamy S, et al. Evaluation of metabolic and immunological changes in streptozotocin-nicotinamide induced diabetic rats. Cell Immunol 2014; 289: 145-9.

22. Reitman S, Frankel SA. A colorimetric method for the determination of serum glutamic oxaloacetic and glutamic pyruvic transaminases. Am J Clin Pathol 1957; 28 : 56-63.

23. Doumas BT, Bayse D, Borner K, Cart RJ, Peters T, Schaffer $R$. A candidate reference method for the determination of total protein in serum: development and validation. Clin Chem 1981; 27: 1642-50.

24. Diniz YS, Rocha KK, Souza GA, Galhardi CM, Ebaid GM, Rodrigues HG. Effects of $\mathrm{N}$-acetylcysteine on sucrose-rich diet-induced hyperglycaemia, dyslipidemia and oxidative stress in rats. Eur J Pharmacol 2006; 543: 151-7.

25. Werner M, Gabrielson D, Eastman G. Ultramicro determination of serum triglycerides by bioluminescent assay. Clin Chem 1981; 27: 268-71.

26. Allain CC, Poo LS, Chan CSG. Enzymatic determination of total serum cholesterol. Clin Chem 1974; 20: 470-5.

27. Li Q, Wu J, Guo D, Cheng H, Chen S, Chan S. Suppression of diet-induced hypercholesterolemia by scutellarin in rats. Plantamedica 2009; 75: 1203-8.

28. Bancroft J, Marilyn G. Theory and Practice of Techniques. Sixth Edition. Churchill Livingstone, London 2007; 164-5.

29. Saukkonen JJ, Cohn DL, Jasmer RM, Schenker S, Jereb JA. An official ATS statement: hepatotoxicity of antituberculosis therapy. Am J Respir Crit Care Med 2006; 174: 935-52.

30. Abou-Seif MA, Youssef A. Evaluation of some biochemical changes in diabetic patients. Clin Chim Acta 2004; 346: 161-70.

31. Deveaud C, Beauvoit B, Reynaud A, Bonnet J. Sitespecific reduction of oxidative and lipid metabolism in adipose tissue of 3-azido-3-deoxythymidine-treated rats. Antimicrob Agents Chemother 2006; 51: 583-90.

32. Afolayan AJ, Yakubu MT. Effects of Bulbine natalensis Baker stem extract on the functional indices and histology of the liver and kidney of male Wistar rats. J Med Food 2009; 12: 814-20.

33. Sulkowski MS, Mehta SH, Chaisson RE, Thomas RE, Moore DL, Richard D. Hepatotoxicity associated with protease inhibitor-based antiretroviral regimens with or without concurrent ritonavir. AIDS 2004; 18: 2277-84.

34. Boone L, Meyer M, Cusick P, et al. Selection and interpretation of clinical pathology indicators of hepatic injury in preclinical studies. Vet Clin Pathol 2005; 34: 182-8.

35. Thapa BR, Walia A. Liver function tests and their interpretation. Indian J Pediatr 2007; 74: 663-71.

36. Ramana KV. Effect of highly active antiretroviral therapy (HAART) on human immunodeficiency virus disease pathogenesis and progression. Am J Public Health Res 2014; 2: 68-74.

37. Kumarappan CT, Thilagam E, Vijayakumar M, Mandal SC. Modulatory effect of polyphenolic extracts of Ichnocarpus frutescens on oxidative stress in rats with experimentally induced diabetes. Indian J Med Res 2012; 136: 815-21.

38. Carr A, Workman C, Carey D, et al.; The Rosey Investigators. No effect of rosiglitazone for HIV-1 lipoatrophy: 
a randomised, double-blind, placebo controlled trial. Lancet 2004; 363: 429-38.

39. Feeney ER, Mallon PGW. HIV and HAART-associated dyslipidemia. Open Cardiovasc Med J 2011; 5: 49-63.

40. Mallal SA, John M, Moore CB, James IR, McKinnon EJ. Contribution of nucleoside analogue reverse transcriptase inhibitors to subcutaneous fat wasting in patients with HIV infection. AIDS 2000; 14: 1309-16.

41. Fouad A, Ahmed MS, Abdel-Lattife, et al. The role of chitosan and wheat germ as antidiabetic substances in diabetic rats. RJPBCS 2014; 5: 457-69.

42. Sahib N, Farooq A, Anwarul H, Khalid M. Coriandrum sativum: a potential source of high-value components for functional foods and nutraceuticals - a review. Phytother Res 2012; 10: 1002-20.

43. Wyatt CM, Morgello S, Katz-Malamed R. The spectrum of kidney disease in patients with AIDS in the era of antiretroviral therapy. Kidney Int 2009; 75: 428-34.

44. Guneli E, Tugyan K, Ozturk H, Gumustekin M, Cilaker S, Uysal N. Effect of melatonin on testicular damage in streptozotocin-induced diabetes rats. Eur Surg Res 2008; 40: 354-60.

45. Azu OO, Naidu ECS, Naidu JS, Masia T, Nzemande NF. Testucular histomorphologic and stereological alterations following short-term treatment with highly active antiretroviral drugs (HAART) in an experimental animal model. Andrology 2014; 2: 772-9.

46. Grundy SM, Brewer HB Jr, Cleeman J, Smith SC Jr, Lenfant C. Definition of metabolic syndrome: report of the National Heart, Lung, and Blood Institute/American Heart Association conference on scientific issues related to definition. Circulation 2004; 109: 433-8.

47. Amin A, Mustafa IO, Folarin RO, Onanuga IO, Ibrahim RB, Balogun WG. Effect of Nigerian propolis on glycaemia, lipid profile, and oxidative stress markers in alloxan-induced diabetic rats. Pharmacol Online 2013; 2: 149-58.

48. Mills E, Cooper C, Seely D, Kanfer I. African herbal nedicines in the treatment of HIV: hypoxis and Sutherlandia. Overview of evidence and pharmacology. Nutr J 2005 4: 19.

49. Charles A, Patrick JB, Collen MM, Bernd R. Inhibition of major drug metabolizing CYPs by common herbal medicines used by HIV/AIDS patients in Africa - implications for herb-drug interactions. Drug Metab Lett 2014; 7: 83-95. 